\title{
Fractional Central Differences and Derivatives
}

\author{
MANUEL DUARTE ORTIGUEIRA \\ UNINOVA and Department of Electrical Engineering, Faculdade de Ciências e Tecnologia da \\ Universidade Nova de Lisboa, Campus da FCT da UNL, Quinta da Torre, 2825 - 114 Monte da \\ Caparica,Portugal (mdo@fct.unl.pt)
}

(Received 17 November 2005; accepted 4 October 2006)

\begin{abstract}
Fractional central differences and derivatives are studied in this article. These are generalisations to real orders of the ordinary positive (even and odd) integer order differences and derivatives, and also coincide with the well known Riesz potentials. The coherence of these definitions is studied by applying the definitions to functions with Fourier transformable functions. Some properties of these derivatives are presented and particular cases studied.
\end{abstract}

Keywords: Fractional central difference, fractional central derivative, Grünwald-Letnikov, generalized Cauchy derivative

\section{INTRODUCTION}

In previous work (Ortigueira and Coito, 2004; Ortigueira, 2006a), we proposed a new approach for introducing causal and anti-causal fractional derivatives based on four steps:

1. Use as a starting point the Grünwald-Letnikov forward and backward differences and derivatives.

2. With an integral formulation for the fractional differences, and using the asymptotic properties of the Gamma function, obtain the generalised Cauchy derivative.

3. Compute the integral defining the generalised Cauchy derivative, using the Hankel path to obtain regularised fractional derivatives.

4. Through application of these regularised derivatives to functions via the Laplace transform, the Liouville fractional derivative is obtained.

In other articles (Ortigueira, 2006b,c), we presented a similar procedure for central fractional derivatives. It comprises the following steps:

1. Introduction of the general framework for central differences, considering two cases, which we called type 1 and type 2 . These are generalisations of the usual central differences for even and odd positive orders respectively.

2. Limit computation, as for normal Grünwald-Letnikov derivatives.

3. Suitable integral representations for these differences were introduced. From these representations, we obtained the derivative integrals, using the properties of the Gamma 
function. The integration is performed over two infinite lines that "close at infinity" to form a closed path. Two generalisations of the usual Cauchy derivative definition are obtained, which agree with it when $\alpha$ is an even or an odd positive integer, for type 1 and type 2 , respectively.

4. The computation of these integrals over a path of two straight lines leads to generalisations of the Riesz potentials.

The most interesting feature of the relations obtained lies in the summation formulae for the Riesz potentials.

In this article, we revise this procedure and test the coherence of the proposed framework, by applying them to the complex exponential. The results show that they are suitable for functions with Fourier transform. The formulation agrees also with the work of Okikiolu (1966). Special cases are studied, and some properties presented. The remainder of the article is arranged as follows: In section 2 we present the type 1 and type 2 central differences, and their integral representations. Central derivative definitions are presented in Section 3, and their integral representations obtained, generalising the Riesz potentials. In Section 4, we apply the definitions to the complex exponential to test the coherence of the definitions. The special integer order cases are studied, and some properties of the derivatives are presented. Finally, we present some conclusions.

\section{FRACTIONAL CENTRAL DIFFERENCES AND THEIR INTEGRAL REPRESENTATIONS}

In this article, we consider two types of fractional central difference. In what follows, $\alpha>$ $-1, h \in R^{+}$and $f(t)$ is a complex variable function. We define a type 1 difference as

$$
\Delta_{c_{1}}^{\alpha} f(t)=\sum_{-\infty}^{+\infty} \frac{(-1)^{k} \Gamma(\alpha+1)}{\Gamma(\alpha / 2-k+1) \Gamma(\alpha / 2+k+1)} f(t-k h)
$$

and a type 2 difference (for which we additionally assume that $\alpha$ is non-zero) is defined as

$$
\Delta_{c_{2}}^{\alpha} f(t)=\sum_{-\infty}^{+\infty} \frac{(-1)^{k} \Gamma(\alpha+1) f(t-k h+h / 2)}{\Gamma[(\alpha+1) / 2-k+1] \Gamma[(\alpha-1) / 2+k+1]}
$$

Using the relation (Andrews et al., 1999, p. 123)

$$
\begin{aligned}
& \sum_{-\infty}^{+\infty} \frac{1}{\Gamma(a-k+1) \Gamma(b-k+1) \Gamma(c+k+1) \Gamma(d+k+1)} \\
= & \frac{\Gamma(a+b+c+d+1)}{\Gamma(a+c+1) \Gamma(b+c+1) \Gamma(a+d+1) \Gamma(b+d+1)}
\end{aligned}
$$

which is valid for $a+b+c+d>-1$, it is possible to show that 


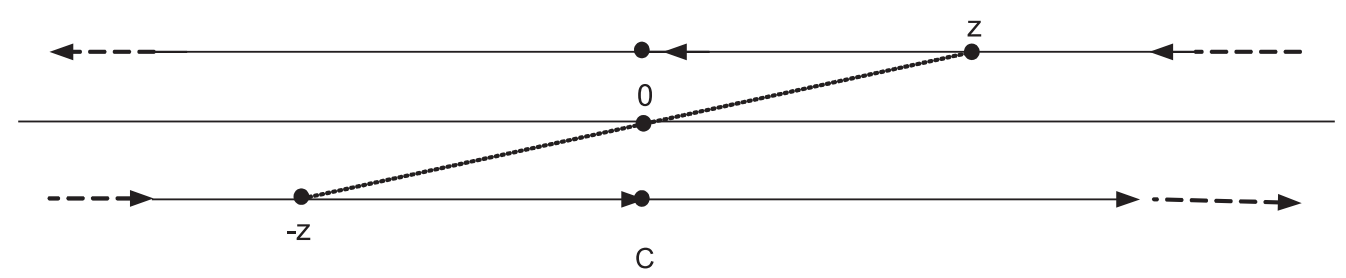

Figure 1. Integration path for integrals (11) and (12).

$$
\Delta_{c_{1}}^{\beta}\left\{\Delta_{c_{1}}^{\alpha} f(t)\right\}=\Delta_{c_{1}}^{\alpha+\beta} f(t)
$$

and

$$
\Delta_{c_{2}}^{\beta}\left\{\Delta_{c_{2}}^{\alpha} f(t)\right\}=-\Delta_{c_{1}}^{\alpha+\beta} f(t)
$$

while

$$
\Delta_{c_{2}}^{\beta}\left\{\Delta_{c_{1}}^{\alpha} f(t)\right\}=\Delta_{c_{2}}^{\alpha+\beta} f(t)
$$

provided that $\alpha+\beta>-1$. In particular, with $\alpha+\beta=0$, relations (4) and (5) show that when $|\alpha|<1$ and $|\beta|<1$ the inverse differences must exist, and can be obtained using formulae (1) and (2). It is worth noting that the zero order type 1 difference as obtained from equation (1)is the identity operator. The zero order type 2 difference will be considered later.

Let us assume that $f(z)$ is analytic in a region of the complex plane that includes the real axis. To obtain integral representations for differences described above, we follow the procedure used by Ortigueira and Coito (Ortigueira and Coito, 2004; Ortigueira, 2006a). We only need to give interpretations to (1) and (2) in terms of the residue theorem. Note that, for the first case, the poles must lie at $n h, n \in Z$. This leads easily to

$$
\Delta_{c_{1}}^{\alpha} f(t)=\frac{\Gamma(\alpha+1)}{2 \pi j h} \int_{C_{c}} f(z+w) \frac{\Gamma\left(\frac{-w}{h}+1\right)}{\Gamma\left(\frac{-w}{h}+\frac{\alpha}{2}+1\right)} \frac{\Gamma\left(\frac{w}{h}\right)}{\Gamma\left(\frac{w}{h}+\frac{\alpha}{2}+1\right)} \mathrm{d} w .
$$

The integrand function has infinitely many poles at every $n h, n \in Z$. The integration path must consist of infinite lines above and below the real axis, closing at infinity. The easiest example is obtained by considering two straight lines near the real axis, one above and the other below (see Figure 1). In the second case, the poles are located at half-integer multiples of $h$, which leads to

$$
\Delta_{c_{2}}^{\alpha} f(t)=\frac{\Gamma(\alpha+1)}{2 \pi j h} \int_{C_{c}} f(z+w) \frac{\Gamma\left(-\frac{w}{h}+\frac{1}{2}\right)}{\Gamma\left(\frac{-w}{h}+\frac{\alpha}{2}+1\right)} \frac{\Gamma\left(\frac{w}{h}+\frac{1}{2}\right)}{\Gamma\left(\frac{w}{h}+\frac{\alpha}{2}+1\right)} \mathrm{d} w .
$$


These integral formulations will be used in the next section to obtain the integral formulae for the central derivatives.

\section{THE FRACTIONAL CENTRAL DERIVATIVES}

To obtain fractional central derivatives we proceed as in previous cases (Samko et al., 1987; Ortigueira and Coito, 2004; Ortigueira, 2006a,b,c): Divide the fractional differences by $h^{\alpha}$ $\left(h \in \mathfrak{R}^{+}\right.$) and let $h \rightarrow 0$. For the first case, and assuming again that $\alpha>-1$, we obtain

$$
\begin{aligned}
D_{c_{1}}^{\alpha} f(t) & =\lim _{h \rightarrow 0} \frac{\Delta_{c_{1}}^{\alpha} f(t)}{h^{\alpha}} \\
& =\lim _{h \rightarrow 0} \frac{\Gamma(\alpha+1)}{h^{\alpha}} \sum_{-\infty}^{+\infty} \frac{(-1)^{k}}{\Gamma(\alpha / 2-k+1) \Gamma(\alpha / 2+k+1)} f(t-k h)
\end{aligned}
$$

which we will call a type 1 fractional central derivative.

For the second case, and still additionally assuming that $\alpha \neq 0$, we obtain the type 2 fractional central derivative as

$$
\begin{aligned}
D_{c_{2}}^{\alpha} f(t) & =\lim _{h \rightarrow 0} \frac{\Delta_{c_{2}}^{\alpha} f(t)}{h^{\alpha}} \\
& =\lim _{h \rightarrow 0} \frac{\Gamma(\alpha+1)}{h^{\alpha}} \sum_{-\infty}^{+\infty} \frac{(-1)^{k} f(t-k h+h / 2)}{\Gamma[(\alpha+1) / 2-k+1] \Gamma[(\alpha-1) / 2+k+1]}
\end{aligned}
$$

Equations (9) and (10) generalise the positive integer order central derivatives to the fractional case, although there should be an extra factor, of $(-1)^{\alpha / 2}$ in the first case and $(-1)^{(\alpha+1) / 2}$ in the second case, which we have removed.

To obtain the integral representations for the derivatives, we commute the limit and integration in (7) and (8). To perform the limit computation inside the integral, we make use of the properties of the gamma function (Henrici, 1974). As shown previously (Ortigueira and Coito, 2004; Ortigueira, 2006b,c), we obtain, for the type 1 case

$$
D_{c_{1}}^{\alpha} f(t)=\frac{\Gamma(\alpha+1)}{2 \pi j} \int_{C_{c}} f(z+w) \frac{1}{(w)_{l}^{\alpha / 2+1}(-w)_{r}^{\alpha / 2}} \mathrm{~d} w
$$

and, for the type 2 derivative

$$
D_{c_{2}}^{\alpha} f(t)=\frac{\Gamma(\alpha+1)}{2 \pi j} \int_{C_{c}} f(z+w) \frac{1}{(w)_{l}^{(\alpha+1) / 2}(-w)_{r}^{(\alpha+1) / 2}} \mathrm{~d} w .
$$


The subscripts " $l$ " and " $r$ " indicate that the power functions have the left and right half real axis (respectively) as branch cut lines. Relations (11) and (12) are generalisations of the usual Cauchy formulae. Choosing a two straight line integration path as shown in Figure 1, we obtain for the type 1 derivative the expression

$$
D_{c_{1}}^{\alpha} f(t)=\frac{1}{2 \Gamma(-\alpha) \cos (\alpha \pi / 2)} \int_{-\infty}^{\infty} f(z-x) \frac{1}{|x|^{\alpha+1}} \mathrm{~d} x
$$

which is the so-called Riesz potential (Samko et al., 1987; Kilbas et al., 2006). Similarly, and as $\alpha$ is not an odd integer, we obtain for the second case

$$
D_{c_{2}}^{\alpha} f(t)=-\frac{1}{2 \Gamma(-\alpha) \sin (\alpha \pi / 2)} \int_{-\infty}^{\infty} f(z-x) \frac{\operatorname{sgn}(x)}{|x|^{\alpha+1}} \mathrm{~d} x
$$

which is the modified Riesz potential (Samko et al., 1987). Both potentials (13) and (14) have been studied by Okikiolu (1966). These are essentially convolutions of a given function with two acausal (that is, neither causal nor anti-causal) operators, and are suitable for dealing with functions defined in $\Re$, and that are not necessarily equal to zero at $\pm \infty$. In particular, they must be suitable for dealing with stationary stochastic processes (Ortigueira and Batista, 2006).

\section{COHERENCE OF THE DEFINITIONS}

\subsection{Type 1 Derivative}

We propose to test the coherence of the results by considering functions with Fourier transformable functions. To perform this study, we will examine the behaviour of the defined derivatives for $f(t)=\mathrm{e}^{-j \omega t}, t, \omega \in \mathfrak{R}$. In the following we will consider non-integer orders greater than -1 . We start by considering the type 1 derivative. From (1) we obtain

$$
\Delta_{c_{1}}^{\alpha} \mathrm{e}^{j \omega t}=\mathrm{e}^{-j \omega t} \sum_{-\infty}^{+\infty} \frac{(-1)^{n} \Gamma(\alpha+1)}{\Gamma(\alpha / 2-n+1) \Gamma(\alpha / 2+n+1)} \mathrm{e}^{j \omega n h}
$$

where we recognize the discrete-time Fourier transform of $R_{b}(n)$ (which is, in purely mathematical terms a Fourier series with $R_{b}(n)$ as coefficients), given by

$$
R_{b}(n)=\frac{(-1)^{n} \Gamma(\alpha+1)}{\Gamma(\alpha / 2-n+1) \Gamma(\alpha / 2+n+1)} .
$$

This function is the discrete autocorrelation of

$$
h_{n}=\frac{(-\alpha / 2) n}{n !} u_{n}
$$


where $u_{n}$ is the discrete unit step Heaviside function (Ortigueira, 2000). As the discrete-time Fourier transform of $h_{n}$ is

$$
H\left(\mathrm{e}^{j \omega}\right)=F T\left[h_{n}\right]=\left(1-\mathrm{e}^{-j \omega h}\right)^{\alpha / 2}
$$

the discrete-time Fourier transform of $R_{b}(n)$ is

$$
\begin{aligned}
S\left(\mathrm{e}^{j \omega}\right) & =\lim _{z \rightarrow \mathrm{e}^{j \omega h}}\left(1-z^{-1}\right)^{\alpha / 2}(1-z)^{\alpha / 2}=\left(1-\mathrm{e}^{-j \omega h}\right)^{\alpha / 2}\left(1-\mathrm{e}^{j \omega h}\right)^{\alpha / 2} \\
& =\left|\mathrm{e}^{j \omega h / 2}-\mathrm{e}^{-j \omega h / 2}\right|^{\alpha}=|2 \sin (\omega h / 2)|^{\alpha} .
\end{aligned}
$$

Thus,

$$
|2 \sin (\omega h / 2)|^{\alpha}=\sum_{-\infty}^{+\infty} \frac{(-1)^{n} \Gamma(\alpha+1)}{\Gamma(\alpha / 2-n+1) \Gamma(\alpha / 2+n+1)} \mathrm{e}^{j \omega n h}
$$

and we can then write

$$
\Delta_{c_{1}}^{\alpha} \mathrm{e}^{-j \omega t}=\mathrm{e}^{-j \omega t}|2 \sin (\omega h / 2)|^{\alpha}
$$

It follows that there is a linear system with a frequency response given by

$$
H_{\Delta 1}(\omega)=|2 \sin (\omega h / 2)|^{\alpha}
$$

that acts on a signal giving its type 1 central fractional difference. Dividing equation (22) by $h^{\alpha}\left(h \in \mathfrak{R}^{+}\right)$and computing the limit as $h \rightarrow 0$, we get

$$
H_{D 1}(\omega)=|\omega|^{\alpha}
$$

As $\alpha$ is not an even integer,

$$
|\omega|^{\alpha}=\lim _{h \rightarrow 0} \frac{1}{h^{\alpha}} \sum_{-\infty}^{+\infty} \frac{(-1)^{n} \Gamma(\alpha+1)}{\Gamma(\alpha / 2-n+1) \Gamma(\alpha / 2+n+1)} \mathrm{e}^{j \omega n h}
$$

(valid for $\alpha>-1$ ). The inverse Fourier transform of $|\omega|^{\alpha}$ is given (Okikiolu,1966) by

$$
F T^{-1}\left[|\omega|^{\alpha}\right]=\frac{1}{2 \Gamma(-\alpha) \cos (\alpha \pi / 2)}|t|^{-\alpha-1}
$$

and we obtain the impulse response

$$
h_{D 1}(t)=\frac{1}{2 \Gamma(-\alpha) \cos (\alpha \pi / 2)}|t|^{-\alpha-1}
$$


leading to

$$
D_{c_{1}}^{\alpha} f(t)=\frac{1}{2 \Gamma(-\alpha) \cos (\alpha \pi / 2)} \int_{-\infty}^{+\infty} f(\tau)|t-\tau|^{-\alpha-1} \mathrm{~d} \tau
$$

which coincides with (13). Relations (16) and (17) allow us to conclude that the type 1 central derivative is equivalent to the application of the $\alpha / 2$ order forward (or backward) derivative twice: Once with increasing time, and then with reverse time.

\subsection{Type 2 Derivative}

A similar procedure allows us to obtain

$$
\Delta_{c_{2}}^{\alpha} \mathrm{e}^{-j \omega t}=\mathrm{e}^{-j \omega t} \mathrm{e}^{-j \omega h / 2} \sum_{-\infty}^{+\infty} \frac{(-1)^{k} \Gamma(\alpha+1)}{\Gamma[(\alpha+1) / 2-k+1] \Gamma[(\alpha-1) / 2+k+1]} \mathrm{e}^{j \omega k h} .
$$

In order to maintain the coherence with the usual definition of the discrete-time Fourier transform, we change the summation variable, obtaining

$$
\Delta_{c_{2}}^{\alpha} \mathrm{e}^{-j \omega t}=\mathrm{e}^{-j \omega t} \mathrm{e}^{-j \omega h / 2} \sum_{-\infty}^{+\infty} \frac{(-1)^{k} \Gamma(\alpha+1)}{\Gamma[(\alpha+1) / 2+k+1] \Gamma[(\alpha-1) / 2-k+1]} \mathrm{e}^{-j \omega k h} .
$$

The coefficients of the above Fourier series are the cross-correlations $R_{b c}(k)$ between

$$
h_{n}=\frac{(-a)_{n}}{n !} u_{n}
$$

and

$$
g_{n}=\frac{(-b)_{n}}{n !} u_{n}
$$

where $a=(\alpha+1) / 2$ and $b=(\alpha-1) / 2$. Let $S_{b c}\left(\mathrm{e}^{j \omega}\right)$ be the discrete-time Fourier transform of the cross-correlation $R_{b c}(k)$ :

$$
S_{b c}\left(\mathrm{e}^{j \omega}\right)=F T\left[R_{b c}(k)\right] .
$$

As $R_{b c}(k)$ is a correlation, we conclude that $S_{b c}\left(\mathrm{e}^{j \omega}\right)$ is given by

$$
\begin{aligned}
S_{b c}\left(\mathrm{e}^{j \omega}\right) & =\lim _{z \rightarrow \mathrm{e}^{j} \omega h}\left(1-z^{-1}\right)^{(\alpha+1) / 2}(1-z)^{(\alpha-1) / 2} \\
& =\left(1-\mathrm{e}^{-j \omega h}\right)^{(\alpha+1) / 2}\left(1-\mathrm{e}^{j \omega h}\right)^{(\alpha+1) / 2}\left(1-\mathrm{e}^{j \omega h}\right)^{-1} .
\end{aligned}
$$


Therefore, we can write

$$
\Delta_{c_{2}}^{\alpha} \mathrm{e}^{-j \omega t}=\mathrm{e}^{j \omega t}|2 \sin (\omega h / 2)|^{\alpha+1}[2 j \sin (\omega h / 2)]^{-1} .
$$

Thus, there is a linear system with a frequency response given by

$$
H_{\Delta 2}(\omega)=|2 \sin (\omega h / 2)|^{\alpha+1}[2 j \sin (\omega h / 2)]^{-1}
$$

that acts on a signal giving its fractional central difference. We can also write

$$
\begin{aligned}
& |2 \sin (\omega h / 2)|^{\alpha+1}[2 j \sin (\omega h / 2)]^{-1} \\
= & \sum_{-\infty}^{+\infty} \frac{(-1)^{k} \Gamma(\alpha+1)}{\Gamma[(\alpha+1) / 2+k+1] \Gamma[(\alpha-1) / 2-k+1]} \mathrm{e}^{-j \omega k h} .
\end{aligned}
$$

Dividing (36) by $h^{\alpha}\left(h \in \mathfrak{R}^{+}\right)$and computing the limit as $h \rightarrow 0$, we get

$$
H_{D 2}(\omega)=-j|\omega|^{\alpha} \operatorname{sgn}(\omega)
$$

As

$$
j \frac{\mathrm{d}|\omega|^{\alpha+1}}{\mathrm{~d} \omega}=j(\alpha+1)|\omega|^{\alpha} \operatorname{sgn}(\omega)
$$

we can obtain from equation (26) using a well known property of the Fourier transform:

$$
h_{D 2}(t)=\frac{-\operatorname{sgn}(t)}{(\alpha+1) 2 \Gamma(-\alpha-1) \cos [(\alpha+1) \pi / 2]}|t|^{-\alpha-1}
$$

or, using the properties of the gamma function

$$
h_{D 2}(t)=-\frac{\operatorname{sgn}(t)}{2 \Gamma(-\alpha) \sin (\alpha \pi / 2)}|t|^{-\alpha-1}
$$

and as previously:

$$
D_{c_{1}}^{\alpha} f(t)=-\frac{1}{2 \Gamma(-\alpha) \sin (\alpha \pi / 2)} \int_{-\infty}^{+\infty} f(\tau)|t-\tau|^{-\alpha-1} \operatorname{sgn}(t-\tau) \mathrm{d} \tau .
$$

Relations (30), (31), and (32) allow us to conclude that the type 2 central derivative is equivalent to the application of the forward (or backward) derivative twice: Once with increasing time, and order $(\alpha+1) / 2$, and then with reverse time and order $(\alpha-1) / 2$.

It is interesting to note that combining (23) with (37) to give 


$$
H_{D}(\omega)=H_{D 1}(\omega)+j H_{D 2}(\omega)
$$

we obtain a function that is null for $\omega<0$. This means that the operator defined by equation (37) is the Hilbert transform of that defined in equation (23), and the corresponding "analytic" derivative is given by the convolution of the function at hand with the operator

$$
h_{D}(t)=\frac{|t|^{-\alpha-1}}{2 \Gamma(-\alpha) \cos (\alpha \pi / 2)}-j \frac{|t|^{-\alpha-1} \operatorname{sgn}(t)}{2 \Gamma(-\alpha) \sin (\alpha \pi / 2)} .
$$

This is formally similar to the Riesz-Feller potentials (Samko et al., 1987).

\subsection{Integer Order Cases}

Consider $\alpha=2 N, N \in Z^{+}$, for a type 1 difference. From this, we obtain

$$
\Delta_{c_{1}}^{2 N} f(t)=\sum_{-N}^{+N} \frac{(-1)^{k}(2 N) !}{(N-k) !(N+k) !} f(t-k h)
$$

which can be rewritten as

$$
\Delta_{c_{1}}^{2 N} f(t)=\sum_{-N}^{+N}(-1)^{k}\left(\begin{array}{c}
2 N \\
N-k
\end{array}\right) f(t-k h) .
$$

Other than a factor of $(-1)^{N}$, this is the current $2 N$ order central difference. With $N=0$, we obtain $f(t)$. Similarly, if $\alpha$ is odd $(\alpha=2 N+1)$, the type 2 difference is equal to the current central difference, except for a factor of $(-1)^{N+1}$. In fact, we now have

$$
\Delta_{c_{2}}^{2 N+1} f(t)=\sum_{-N}^{N+1} \frac{(-1)^{k}(2 N+1) ! f(t-k h+h / 2)}{(N+1-k) !(N+k) !}
$$

and

$$
\Delta_{c_{2}}^{2 N+1} f(t)=\sum_{-N}^{N+1}(-1)^{k}\left(\begin{array}{c}
2 N+1 \\
N+k
\end{array}\right) f(t-k h+h / 2) .
$$

In particular, with $N=0$, we obtain

$$
\Delta_{c_{2}}^{1} f(t)=f(t+h / 2)-f(t-h / 2) .
$$

It is interesting to consider the central type 1 difference (or derivative) with $\alpha=2 M+1$ and the type 2 with $\alpha=2 M$. For the first, $\alpha / 2$ is non-integer, and we can use equations (19) to (24). However, they are difficult to manipulate; we found it better to use equation (25), although it is necessary to avoid the product $\Gamma(-\alpha) \cdot \cos (\alpha \pi / 2)$, as the first factor is $\infty$ 
and the second is zero. To solve the problem, we use the reflection formula for the gamma function to obtain

$$
\frac{1}{2 \Gamma(-\alpha) \cos (\alpha \pi / 2)}=-\frac{\Gamma(\alpha+1) \sin (\alpha \pi / 2)}{\pi}
$$

and get a factor equal to $-\frac{(2 M+1) !(-1)^{M}}{\pi}$, when $\alpha=2 M+1$. Finally, we obtain (Kilbas et al., 2006)

$$
F T^{-1}\left[|\omega|^{2 M+1}\right]=-\frac{(2 M+1) !(-1)^{M}}{\pi}|t|^{-2 M-2}
$$

and the corresponding impulse response as

$$
h_{D 1}(t)=-\frac{(2 M+1) !(-1)^{M}}{\pi}|t|^{-2 M-2} .
$$

For the second case, with $\alpha=2 M$, we use equation (37). As above, we have the product $\Gamma(-\alpha) \cdot \sin (\alpha \pi / 2)$. Using the reflection formula of the gamma function, we obtain

$$
-\frac{1}{2 \Gamma(-\alpha) \sin (\alpha \pi / 2)}=\frac{\Gamma(\alpha+1) \cos (\alpha \pi / 2)}{\pi}
$$

and get a factor $\frac{(2 M) !(-1)^{M}}{\pi}$. We then obtain (Kilbas et al., 2006)

$$
F T^{-1}\left[|\omega|^{2 M} \operatorname{sgn}(\omega)\right]=\frac{\operatorname{sgn}(t)(2 M) !(-1)^{M}}{\pi}|t|^{-2 M-1}
$$

and

$$
h_{D 2}(t)=\frac{\operatorname{sgn}(t)(2 M) !(-1)^{M}}{\pi}|t|^{-2 M-1} .
$$

As we can see, equations (48) and (52) allow us to generalize the Riesz potentials for positive integer orders. However, there is no inverse for these equations. It is interesting to study the situation defined by $\alpha=0$ in the type 2 derivative. From equations (14) and (50), we obtain

$$
D_{c_{2}}^{0} f(t)=\frac{1}{\pi} \int_{-\infty}^{\infty} f(z-x) \frac{1}{x} \mathrm{~d} x
$$

which is the Hilbert transform of $f(t)$.

These results allow us to conclude that:

1. Both type 1 (9) and type 2 (10) derivatives are defined and meaningful for real orders greater than -1 .

2. When the order is an even integer, a type 1 derivative is equal to the common derivative with the same order, other than the sign. The same is true for a type 2 derivative if the order is an odd integer. 
3. For the same orders, these derivatives cannot be expressed by the Riesz potentials (13) and (14), because the factors before the integrals are zero.

\subsection{Other Properties of the Central Derivatives}

From relations (4), (5), and (6) we can easily obtain

$$
D_{c_{1}}^{\beta}\left\{D_{c_{1}}^{\alpha} f(t)\right\}=D_{c_{1}}^{\alpha+\beta} f(t)
$$

and

$$
D_{c_{2}}^{\beta}\left\{D_{c_{2}}^{\alpha} f(t)\right\}=-D_{c_{1}}^{\alpha+\beta} f(t)
$$

while

$$
D_{c_{2}}^{\beta}\left\{D_{c_{1}}^{\alpha} f(t)\right\}=D_{c_{2}}^{\alpha+\beta} f(t)
$$

retaining the condition that $\alpha+\beta>-1$. From this, we can conclude that if $|\alpha|<1$ and $|\beta|<1$, the fractional derivative has always an inverse, and also that we can generate the Hilbert transform of a given function with derivations of different types and symmetric orders.

\section{CONCLUSIONS}

In this article, we have introduced a general framework for defining fractional central differences, and considered two cases that are generalisations of the usual central differences. These new differences lead to central derivatives similar to the usual Grünwald-Letnikov ones. For those differences, we presented integral representations, from which we obtained the derivative integrals, similar to Cauchy derivatives, using the properties of the Gamma function. The computation of those integrals led to generalisations of the Riesz potentials. The most interesting feature lies in the summation formulae for the Riesz potentials. To test the coherence of the proposed definitions we applied them to a complex exponential. The results show that they are suitable for functions with Fourier transforms. Some properties of these derivatives were presented.

\section{REFERENCES}

Andrews, G. E., Askey, R., and Roy, R., 1999, Special Functions, Cambridge University Press, Cambridge, UK. Henrici, P., 1974, Applied and Computational Complex Analysis, John Wiley \& Sons, New York, Vol. 1, pp. 270271.

Kilbas, A. A., Srivastava, H. M., and Trujillo, J. J., 2006, Theory and Applications of Fractional Differential Equations, Elsevier, Amsterdam, The Netherlands.

Okikiolu, G. O., 1966, "Fourier transforms of the operator $\mathrm{H}_{\alpha}$," Proceedings of the Cambridge Philosophy Society 62, 73-78. 
Ortigueira, M. D., 2000, "Introduction to fractional signal processing. Part 2: Discrete-time systems," IEE Proceedings on Vision, Image and Signal Processing 147(1), 71-78.

Ortigueira, M. D. and Coito, F., 2004, "From differences to differintegrations," Fractional Calculus \& Applied Analysis 7(4).

Ortigueira, M. D., 2006, “A coherent approach to non integer order derivatives,” Signal Processing 86(10), special section: Fractional calculus applications in signals and systems, 2505-2515.

Ortigueira, M. D., 2006, "Fractional centred differences and derivatives," in Proceedings of the $2^{\text {nd }}$ IFAC Workshop on Fractional Differentiation and its Applications, Porto, Portugal, July 19-21.

Ortigueira, M. D., 2006, "Riesz potentials and inverses via centred derivatives," International Journal of Mathematics and Mathematical Sciences 2006, 1-12.

Ortigueira, M. D. and Batista, A. G., 2006, "On the fractional derivative of stationary stochastic processes," in Proceedings of the $8^{\text {th }}$ International Conference on Computational Structures Technology and the $5^{\text {th }}$ International Conference on Engineering Computational Technology, Las Palmas de Gran Canaria, Spain, September 12-15.

Podlubny, I., 1999, Fractional Differential Equations, Academic Press, New York.

Samko, S. G., Kilbas, A. A., and Marichev, O. I., 1987, Fractional Integrals and Derivatives-Theory and Applications, Gordon and Breach Science Publishers, New York. 